\title{
Acquired thrombotic thrombocytopenic purpura: Caplacizumab as a novel therapeutic option
}

Miguel Relvas ${ }^{1,2}$, Maria Lume ${ }^{3}$, Fernando Friões $3,4,5$, Luís Coentrão ${ }^{1,2,5}$

\footnotetext{
${ }^{1}$ Nephrology Department, Centro Hospitalar e Universitário de São João, Oporto, Portugal

${ }^{2}$ Nephrology \& Infectious Diseases R\&D, i3S - Instituto de Investigação e Inovação em Saúde da Universidade do Porto, Oporto, Portugal

3 Intermediate Care Unit, Department of Internal Medicine, Centro Hospitalar e Universitário de São João, Oporto, Portugal

${ }^{4}$ Cardiovascular Research and Development Center (UnIC), Universidade do Porto, Oporto, Portugal

${ }^{5}$ Faculty of Medicine, Universidade do Porto, Oporto, Portugal
}

\section{Dear editor,}

Thrombotic thrombocytopenic purpura (TTP) is a medical emergency among primary thrombotic microangiopathies that relates to decreased activity of ADAMTS13, a metalloprotease responsible for the breakdown of von Willebrand factor (VWF). Most cases result from the formation of inhibitory autoantibodies (acquired TTP - aTTP). ${ }^{1}$
Plasma exchange (PEX) is the cornerstone of aTTP treatment. Standard treatment typically also includes corticosteroids and rituximab. ${ }^{2,3}$ Caplacizumab is a humanized monoclonal antibody fragment that binds to vWF and blocks its interaction with platelets, consequently reducing microthrombi formation. It is currently recommended as an add-on to the standard approach in severe and refractory cases of aTTP. ${ }^{4}$

\section{Figure 1}

(A) Platelet count progression during hospitalization; (B) ADAMTS13 activity progression after institution of appropriate therapy.
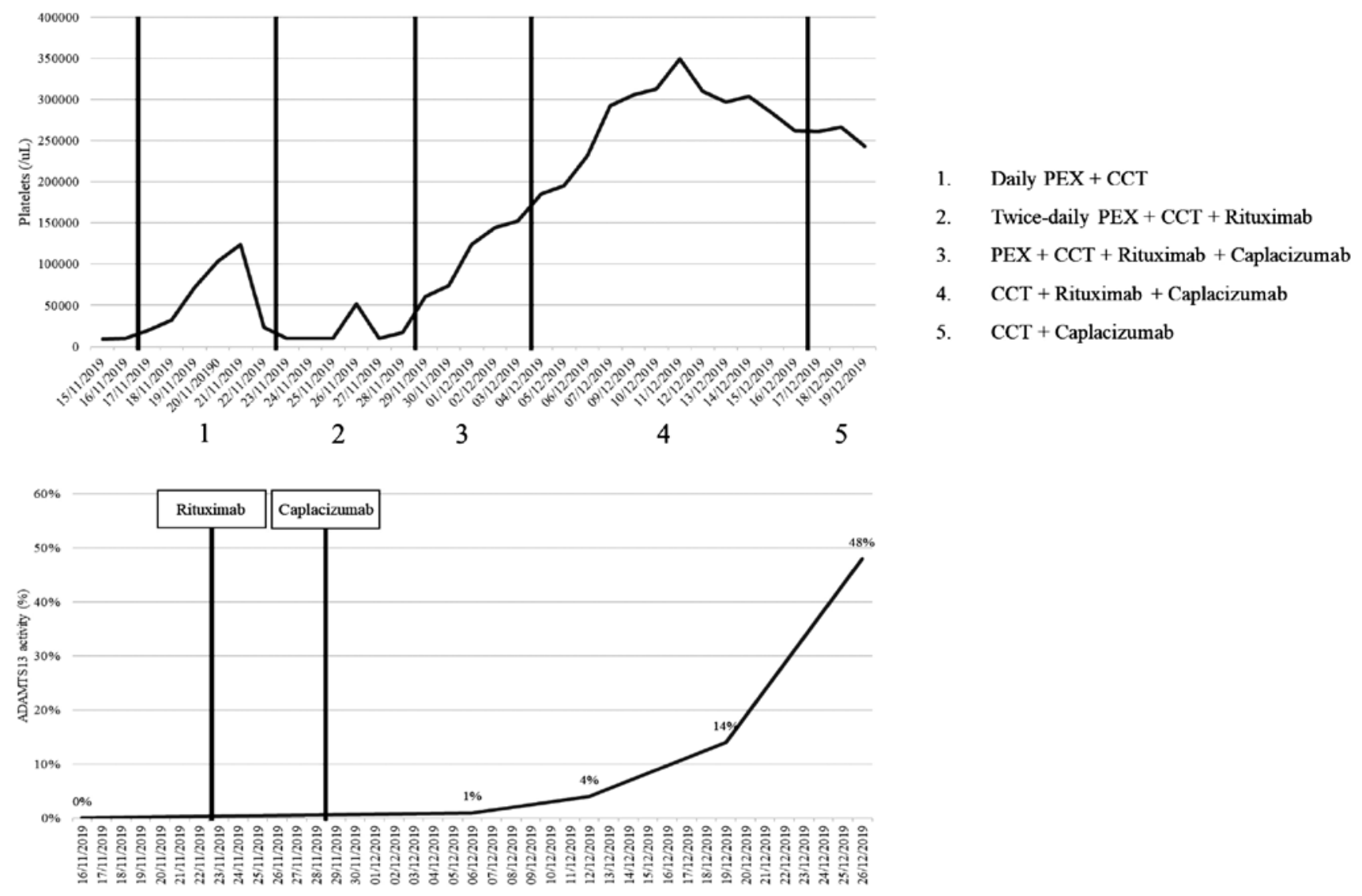
In late 2019, a 36-year-old woman with personal history of Sjögren syndrome presented to our hospital with complaints of asthenia, epistaxis and disperse petechiae. Laboratory testing revealed normocytic normochromic anemia, severe thrombocytopenia, no schizocytes, nonconjugated hyperbilirubinemia and significant elevation of lactate dehydrogenase with a negative Coombs test. Coagulation tests and autoimmune screening were both unremarkable. After an initial dose of methylprednisolone, the patient was discharged from the hospital under prednisolone with instructions to return after 48 hours for clinical reassessment.

Returning to the hospital, the patient referred worsening complaints of asthenia and a spontaneous hematoma in the left frontal region. Laboratory testing showed aggravated anemia, sustained severe thrombocytopenia, and de novo schizocytes. Coombs testing was again negative. Viral serologies (for HIV, HBV, and HCV) were all negative and coagulation tests remained normal.

Due to clinical and analytic deterioration, the patient was hospitalized for further care and investigation. She worsened progressively over the first 24 hours, with aggravated anemia, language alterations and motor dyspraxia, despite a normal brain CT scan. A presumptive diagnosis of aTTP was made - blood samples were collected for ADAMTS13 testing and the patient started daily PEX, maintaining daily prednisolone $(1 \mathrm{mg} / \mathrm{kg})$.

Despite slight initial improvement, the patient presented with relapsed severe thrombocytopenia after five days. Thus, PEX was intensified to a twice-daily scheme and rituximab was introduced (375 $\mathrm{mg} / \mathrm{m} 2$ - once weekly for four weeks). ADAMTS13 results were then made available, confirming aTTP diagnosis - severely deficient activity (0\%) with high anti-ADAMTS13 titers (93 U).
Considering the patient's suboptimal response to standard treatment, caplacizumab was introduced, resulting in a rapid increase of the platelet count along with symptomatic resolution. PEX was suspended five days after caplacizumab introduction. The patient was hospitalized for 35 days in total to complete rituximab treatment under surveillance. She progressed favorably and was discharged still under caplacizumab and prednisolone. Figure 1 represents the progression of the patient's platelet count and ADAMTS13 activity.

Associating caplacizumab to standard therapy proved essential in this case. To our knowledge, this was the first successful use of this agent in Portugal. At the time of writing, only two patients have benefitted from caplacizumab treatment in our country, with its use currently limited to refractory cases. Optimal incorporation of this drug into clinical practice is yet to be determined, but its use must be considered in severe forms of aTTP and in patients presenting with refractory disease.

Disclosure of potential conflicts of interest: none declared

\section{References}

1. Arnold DM, Patriquin CJ, Nazy I. Thrombotic microangiopathies: a general approach to diagnosis and management. Cmaj. 2017;189(4):E153-E159.

2. Rock G. The management of thrombotic thrombocytopenic purpura in 2005. Semin Thromb Hemost. 2005;31(6):709-716.

3. Cuker A. Adjuvant rituximab to prevent TTP relapse. Blood. 2016;127(24):2952-2593.

4. le Besnerais M, Veyradier A, Benhamou Y, Coppo P. Caplacizumab: a change in the paradigm of thrombotic thrombocytopenic purpura treatment. Expert Opin Biol Ther. 2019;19(11):1127-1134.

\section{Correspondence to:}

Miguel Relvas, MD

Nephrology Department, Centro Hospitalar e Universitário de São João E-mail: mic21892@gmail.com 